\title{
Comprendiendo la sustentabilidad desde un punto de vista exergético en sistemas adaptativos complejos
}

\section{Understanding sustainability from an exergetic frame in complex adaptive systems}

\author{
Glenn Alonso Aguilar Hernández. \\ Universidad de Costa Rica. Escuela de Ingeniería Agrícola \\ glenn.aguilar@ucr.ac.cr
}

Recibido: 9 de febrero 2016

Aceptado: 8 de agosto 2016

\begin{abstract}
Resumen
El concepto de sustentabilidad ha sido utilizado en un extenso número de áreas de estudio, lo cual ha implicado múltiples interpretaciones sobre la definición de sustentabilidad. Este documento desarrolló de manera crítica un marco conceptual para la definición general sobre sustentabilidad a partir de propiedades termodinámicas que son aplicadas a sistemas adaptativos complejos. Primeramente, se hizo referencia a los orígenes de la percepción sobre desarrollo sustentable y la limitación en su aplicación para poder analizar la interacción entre un sistema y su entorno. Posteriormente, se tomó como base las propiedades de un sistema adaptativo complejo y se definió de forma conceptual cómo puede ser afectado un sistema por la restricción de recursos e irreversibilidad de los procesos. Esto permitió dar una comprensión sobre el sistema adaptativo complejo utilizando la primera y segunda ley de la termodinámica, en las cuales se desarrolló un marco conceptual para definir la sustentabilidad de un sistema. Del análisis emergió el factor exergético como un aspecto fundamental para determinar la sustentabilidad de un sistema adaptativo complejo. Con ello se presentan oportunidades para desarrollar estudios en múltiples sistemas como los económicos, sociales y ambientales, en donde se requiere la interdisciplinariedad para generar un marco integral en la aplicación del concepto de sustentabilidad.
\end{abstract}

\section{Palabras clave}

Desarrollo sostenible, termodinámica-modelos matemáticos, termodinámica- mediciones, protección del medio ambiente, gestión ambiental.

\begin{abstract}
The sustainability concept has been applied in a number of fields, which has generated different definitions of sustainability. The following paper has critically developed a conceptual framework for defining sustainability based on thermodynamics properties, which can be applied to complex adaptive systems (CAS). First, there is a description of the general perception of sustainable development and the conceptual limitations to evaluate the interaction between a
\end{abstract}


system and its surroundings. Then, the CAS's properties and the impact of resource restriction were critically evaluated and irreversibility on the CAS's conditions was defined. This brought a broad understanding about the effects on CAS, based on the first and second law of thermodynamics. From this analysis, the exergetic aspect emerged as a fundamental property for defining sustainability in the CAS's context. This shows opportunities for developing studies based on thermodynamics properties in multiple systems, such as economic, social and ecological fields, which would require an interdisciplinary approach to generate an integral framework on the application of sustainability definition.

\section{Keywords}

Sustainable development, thermodynamics- mathematical modelling, thermodynamics- measurements, environmental protection, environmental management. 


\section{INTRODUCCIÓN}

El concepto de sustentabilidad ha sido aplicado en un amplio número de campos de estudio como parte de las iniciativas para contribuir con la continuidad de las actividades humanas. Esta percepción ha sido punto de discusión a través de la historia, en donde se puede destacar la definición de desarrollo sustentable presentada por la Comisión Mundial sobre Medio Ambiente y Desarrollo en 1987, la cual ha sido base para la actual línea de pensamiento en relación con "no comprometer las necesidades de las futuras generaciones" y que ha representado los fundamentos para el concepto de sustentabilidad utilizado en múltiples áreas de investigación $(1,2)$. Sin embargo, las implicaciones de un "sistema sustentable" muestran diversas interpretaciones que causan un entendimiento erróneo en cuanto a la aplicación del concepto de sustentabilidad $(3,4)$. Asimismo, el concepto de sustentabilidad ha sido desarrollado en ocasiones sin considerar el tipo de sistema a analizar, donde se encuentra un interés por evaluar la durabilidad del sistema adaptativo complejo (CAS por siglas en inglés). Por ejemplo, cuando se toma la definición de sustentabilidad en un contexto de cambio climático, primeramente, hay que comprender que el sistema climático no presenta una tendencia lineal y que hay interacciones entre otros subsistemas que presentan características tanto intrínsecas como extrínsecas, las cuales van a determinar la variabilidad del sistema a largo plazo (5).

Un aporte esencial al concepto de sustentabilidad es brindado a partir de propiedades termodinámicas, que pueden ser expresadas para sistemas adaptativos complejos. Ya se han analizado acercamientos a la definición de sustentabilidad a partir de propiedades termodinámicas tomando en consideración un punto de vista de optimización entrópica, donde se ha concluido la relevancia del uso de propiedades termodinámicas para definir la durabilidad de sistemas complejos debido a la irreversibilidad de ciertos procesos naturales (6). Este punto de vista puede desarrollarse de manera más amplia al considerar aspectos tales como el factor exergético en sistemas adaptativos complejos. La exergía tiene que ser clarificada para un contexto de sistemas abiertos, ya que esta está definida en un marco de procesos reversibles, lo cual restringe su aplicación para analizar el comportamiento de sistemas abiertos donde la irreversibilidad del proceso emerge del sistema (7).

El siguiente artículo tiene como objetivo presentar una revisión de lo que se entiende convencionalmente por desarrollo sustentable y cómo la aplicación de principios termodinámicos puede contribuir al desarrollo de un marco conceptual sobre sustentabilidad para ser aplicado de manera general a sistemas adaptativos complejos.

\section{PERCEPCIÓN CONVENCIONAL SOBRE LA DEFINICIÓN DE SUSTENTABILIDAD}

El explorar el significado de qué es "sustentable" requiere, primeramente, la identificación de interacciones entre sistema-entorno y de cómo evolucionan los parámetros a través del tiempo. Esto, por ejemplo, es claro cuando se trata de definir la sustentabilidad en sistemas sociales y los impactos sobre ecosistemas. De hecho, hay que considerar cómo las percepciones culturales sobre la interacción sociedad-ecosistemas han formado las bases fundamentales para lo que se entiende como desarrollo sustentable de la actualidad (2). Por lo tanto, independientemente de la definición que se adquiera 
sobre sustentabilidad y desarrollo sustentable, deberían incluirse en alguna medida los aspectos que predominan en la interacción sociedad-ecosistemas.

En esta perspectiva, hay que considerar la importancia de los aspectos culturales, los cuales tienen un impacto sobre la forma en que se perciben las interacciones entre sistemas. En el caso de la cultura occidental, se observa una tendencia a la creencia de la superioridad de los seres humanos respecto a otras especies, por lo cual hay un predominio en pensar que el ser humano es un tipo de administrador de los bienes naturales (8). Por otro lado, se encuentran casos en donde la visión de mundo consiste en una coexistencia entre el ser humano y su ambiente, como los que se han observado en comunidades ancestrales en territorio africano y en algunas tribus amazónicas $(2,8)$. A pesar de que la perspectiva de estas tribus es más cercana al principio establecido por la Comisión Mundial sobre Medio Ambiente y Desarrollo, la prevalencia del pensamiento occidental parece ser inconsistente con los esfuerzos por generar un crecimiento de manera continua a largo plazo. Esto ha sido demostrado en las últimas décadas al analizar la influencia antropogénica en los efectos negativos sobre algunos ecosistemas de importancia para la adquisición de los recursos necesarios para la subsistencia de futuras generaciones.

Lo anterior refleja también otro aspecto que ha generado confusión sobre la definición de sustentabilidad, la cual en ocasiones se malinterpreta como un tema meramente ambiental. Se encuentra en ocasiones que la definición de sustentabilidad fue creada desde un punto de vista ecológico, el cual sólo representa un aspecto de la interacción entre sistemas (9). Sin embargo, la sustentabilidad ecológica no menciona otros aspectos claves para la comprensión de sistemas y su durabilidad a través del tiempo. De hecho, esto se debe a que el concepto se expresa en un contexto multi e intra-disciplinario, en donde se ven envueltos diversos temas que tienen que ser resueltos bajo el mismo concepto, por ejemplo, crecimiento económico, demográfico, justicia social y resiliencia ecológica $(9,10)$. Es por esto que algunos investigadores sostienen que no es posible brindar una definición general de sustentabilidad, ya que es un concepto que puede variar dependiendo del contexto o disciplina (4).

A pesar de la complejidad en términos contextuales, es posible llegar a una definición más general del significado de sustentabilidad al basarse en las propiedades de los sistemas. Esto quiere decir que, para establecer el primer paso en la definición general, se requiere tomar en cuenta ciertas características que puedan ser reproducidas en cada sistema analizado y que a su vez sirvan para evaluar la interacción con otros subsistemas. Para el presente caso, estas características deberían de enfocarse en un contexto de sistemas adaptativos complejos debido a la dinámica que presentan las actividades antropogénicas y los flujos en ecosistemas (3).

\section{PROPIEDADES DE SISTEMAS ADAPTATIVOS COMPLEJOS DESDE UN PUNTO DE VISTA TERMODINÁMICO}

Asumiendo que cada sistema en el mundo biofísico comparte características similares que emergen desde dinámicas con múltiples interacciones y su conexión con otros subsistemas, la comprensión de los elementos que constituyen un sistema 
adaptativo complejo (como, por ejemplo, límites abiertos del sistema, la no linealidad, fluctuaciones e impredictibilidad, dinámicas lejos del equilibrio, resiliencia y una tendencia a la auto-organización) es el componente fundamental para identificar los puntos que convergen en una percepción general de la sustentabilidad. Levin (11) explicó las propiedades del sistema abierto, no lineal y fluctuante a través de su enfoque en ecosistemas, donde se observa que la transferencia de materia y energía ocurren simultáneamente en múltiples sistemas bióticos y abióticos. Estos cambian a través del tiempo y, según las presiones externas, pueden generar la continuidad o degradación del ecosistema. Por otro lado, Dietz y sus colegas (12) presentaron cómo la dinámica, lejos del equilibrio, hace que un sistema adaptativo se mantenga en una constante auto-organización que le permite al sistema reorganizar su estructura de manera tal que sea resiliente hacia las nuevas condiciones.

A partir de las propiedades del sistema adaptativo complejo se infiere que los sistemas en este orden no son estáticos y que cada sistema presenta límites en cuanto a recursos y dependencia temporal. Entonces, tanto los recursos en términos materiales-energéticos como la irreversibilidad de los procesos, donde se requiere invertir mayor cantidad de energía para revertir el estado de un sistema, son dos factores que pueden variar las propiedades del sistema adaptativo complejo.

En términos de limitación de recursos, Hardin (13) explicó las razones por las cuales no es posible establecer una solución de carácter técnico para solucionar la carencia de recursos en un contexto de incremento de la demanda por estos recursos, considerando un sistema en equilibrio dinámico. Sin embargo, esta idea no considera que un sistema adaptativo complejo fluctúa a través del tiempo y hay una tendencia a la reorganización, lo cual emerge desde la adaptabilidad del sistema debido a que puede haber trasferencia de material-energía por medio de los límites abiertos del sistema (11). Asimismo, los flujos de materia-energía a través de las fronteras del sistema y su entorno se rigen por la conservación de la materia y energía, que es expresada por la primera ley de la termodinámica (14).

La comprensión de la dependencia temporal es otro aspecto esencial de la complejidad en sistemas adaptativos. Por ejemplo, desde una perspectiva evolutiva biológica, se nota que la escala de tiempo en sistemas no lineales favorece una dinámica lejos del equilibrio (15). Esto implica que un sistema adaptativo complejo puede ser impredecible, pero, a su vez, los eventos ocurren en una sola dirección temporal, por lo cual es clara la irreversibilidad de procesos en un sistema adaptativo complejo. La segunda ley de la termodinámica presenta a la entropía como una propiedad del sistema que muestra la dirección en la ocurre un proceso. Esta se define, para sistemas abiertos, como la suma de la entropía del sistema y la entropía del entorno (o producción de entropía) $(16,17)$. Uno de los factores claves para tomar el elemento entrópico es que este puede cuantificar el impacto realizado por cierta actividad dentro del sistema sobre su entorno, ya que la energía disipada por un sistema va a tener consecuencias directas e irreversibles sobre otros subsistemas y sus alrededores.

Debido a la relación que existe entre sistemas adaptativos complejos y las propiedades termodinámicas, es posible establecer conexiones entre la primera y la segunda ley de la termodinámica que permitan establecer un marco conceptual para los impactos 
de un sistema sobre el entorno. Para ello, el principio de exergía, el cual se refiere al trabajo máximo que puede ser utilizado por un sistema en relación con su entorno, brinda la oportunidad de ser aplicado para múltiples sistemas $(7,18)$. En términos de aplicabilidad, esta propiedad es considerada de mayor relevancia para la evaluación de sistemas adaptativos complejos, ya que el factor exergético es consecuencia de la primera y segunda ley de la termodinámica y cuantifica tanto la cantidad como la calidad de recurso energético a través de un proceso dado $(18,19)$. En general, un sistema de alta exergía presenta mejor aprovechamiento de los recursos (tanto materiales como energéticos) y la reducción de la producción de entropía, generando así un menor impacto hacia su entorno, lo cual implica la capacidad del sistema de coexistir alrededor de otros subsistemas con un mínimo impacto ambiental (20). Como consecuencia, hay una relación entre la exergía de un sistema y las condiciones de su entorno, que puede ser cuantificable a partir de propiedades termodinámicas y que contribuirían a determinar la sustentabilidad de un sistema adaptativo complejo, produciendo así un marco para establecer una definición general de la sustentabilidad desde un punto de vista analítico.

\section{DISCUSIÓN Y CONCLUSIONES}

Considerando las propiedades de los sistemas adaptativos complejos, la sustentabilidad puede ser definida como la capacidad de un sistema complejo para optimizar su eficiencia exergética, de tal manera que al maximizar la utilización de recursos (materiales y energéticos) y reducir la producción de entropía hacia el ambiente, se obtenga el menor impacto de una actividad productiva. Esto ha tenido un alcance a nivel industrial y se han establecido de manera concreta los flujos de exergía a través de un proceso dado (21). Adicionalmente, se muestran aportes donde se desarrolla la exergía para analizar la sustentabilidad de sistemas en un contexto económico y ambiental. Por ejemplo, se pueden mencionar casos de estudio donde se utiliza la exergía como un factor de eficiencia para determinar el impacto ambiental y económico de algunos sistemas; por ejemplo, aplicado a tecnologías de energías limpias (22). Del mismo modo, el concepto de desarrollo sustentable implicaría aquellas prácticas que brinden un incremento en el factor exergético del sistema y que posibilite el desarrollo del mismo a largo plazo. La relevancia de este marco conceptual es que no observa la sustentabilidad como una meta, sino como una relación entre el sistema y sus alrededores en donde se considera la constante interacción entre los sistemas adaptativos complejos y otros subsistemas.

La definición general de sustentabilidad en términos termodinámicos presenta dos consecuencias inmediatas. Primero, las ciencias relacionadas con la sustentabilidad deberían de enfocarse en la conexión entre subsistemas y el ambiente en vez de cerrar la evaluación a un sistema absoluto como si estuviera analizando un sistema aislado. Segundo, es posible aplicar las propiedades termodinámicas, por lo menos desde el punto de vista conceptual, a diversas disciplinas, con lo cual se puede brindar un amplio entendimiento de sistemas económicos y socio-ambientales en relación con la durabilidad de los sistemas abarcando una definición de carácter holístico.

Ya que la definición toma en cuenta leyes universales (tales como la leyes de la termodinámica), entonces es posible aplicar el concepto a cualquier tipo de sistema que 
cumpla con las características de complejidad y adaptabilidad. En este caso, se puede generar un marco para poder desarrollar un criterio sobre la sustentabilidad en sistemas económicos, sociales y ecológicos en donde habría que establecer para cada caso los parámetros que indique el valor exergético. Por ejemplo, en un tema como el cambio climático, en el cual se muestra un interés por migrar a sistemas productivos en donde se reduzca la huella de carbono, se tendría la oportunidad de cuantificar los impactos causados por la emisión gases de efecto invernadero respecto a cadenas de producción considerando la cantidad y la calidad de la energía utilizada para generar cierto producto o servicio. Esto permitía establecer de manera cuantificable cuáles procesos contribuyen a la reducción del impacto ambiental generado a partir del aumento de la temperatura global como producto del incremento de la concentración de gases de efecto invernadero en la atmósfera y a su vez brindar apoyo para la toma de decisiones en relación con cuáles sistemas productivos favorecen la sustentabilidad de los sectores económicos. Expresar matemáticamente estos sistemas podría presentar complicaciones, especialmente al considerar variables sociales, ya que habría que comenzar por elaborar las metodologías que lleven a obtener la cuantificación del valor exergético como punto de comparación e interrelación entre sistemas. Por ejemplo, se podría pensar en la aplicación de la exergía como parte de un Sistema de Cuentas Ambientales y Económicas (SEEA por sus siglas en inglés), el cual se puede utilizar como una representación de la interacción entre las actividades económicas y los recursos energéticos y naturales $(23,24)$. Adaptando los flujos de exergía para índices en Sistemas Cuentas Ambientales y Económicas, se podría lograr un análisis exergético que muestre las repercusiones socioeconómicas de las actividades a evaluar. Esto brinda un espacio de discusión en donde se puede generar la integración de múltiples disciplinas para el desarrollo conceptual de la sustentabilidad que permita a su vez determinar cuándo un sistema puede ser considerado sustentable desde un punto de vista termodinámico analítico.

\section{REFERENCIAS BIBLIOGRÁFICAS}

1. MCJD. Simposio la Costa Rica del año 2000: documentos. San José; 1977.

2. Mebratu D. Sustainability and sustainable development: historical and conceptual review. Environ Impact Assess Rev. 1998;18(6):493-520.

3. Glavič P, Lukman R. Review of sustainability terms and their definitions. J Clean Prod. 2007;15(18):1875-85.

4. Hugé, J., Waas, T., Dahdouh-Guebas, F., Koedam, N., \& Block T. A discourse-analytical perspective on sustainability assessment: interpreting sustainable development in practice. Sus. 2013;8(2):197-8.

5. IPCC. Climate Change 2013 The Physical Science Basis [Internet]. 2013. Disponible en: http:// www.ipcc.ch/pdf/assessment-report/ar5/wg1/WG1AR5_TS_FINAL.pdf

6. Mata-Segreda JF. ¿Qué hay de erróneo con sustentabilidad ambiental? San José; 2006.

7. Mora-Casal RA. Crítica del concepto de exergía. Ingeniería. 2015;25(1):1409-2241.

8. McMichae A, Butler C, Folke C. New Visions for addressing sustainability. Science. 2003;302(5352):1919-20.

9. Lele SM. Sustainable development: a critical review. World Dev. 1991;19(6):607-21. 
10. Harlow J, Golub A, Allenby B. A review of utopian themes in sustainable development discourse. Sustain Dev. 2013;21(4):270-80.

11. Levin SA. Ecosystems and the biosphere as complex adaptive systems. Ecosystems. 1998;1(5):431-6.

12. Dietz T, Ostrom E, Stern PC. The struggle to govern the commons. science. 2003;302(5652):1907-12.

13. Hardin G. The tragedy of the commons. Science. 1968;162(3859):1243-8.

14. Bejan A, Lorente S. The constructal law of design and evolution in nature. Philos Trans R Soc B Biol Sci. 2010;365(1545):1335-47.

15. Qian H. Stochastic physics, complex systems and biology. Quant Biol. 2013;1(1):50-3.

16. Lucia U. Stationary open systems: A brief review on contemporary theories on irreversibility. Phys A Stat Mech its Appl. 2013;392(5):1051-62.

17. Ozawa H, Ohmura A, Lorenz RD, Pujol T. The second law of thermodynamics and the global climate system: a review of the maximum entropy production principle. Rev Geophys. 2003;41(4).

18. Dincer I, Kanoglu M, Rosen MA. Exergy - Energy, Environment, and Sustainable Development. Amsterdam: Elsevier; 2013. 6-10, 59-65.

19. Hepbasli A. A key review on exergetic analysis and assessment of renewable energy resources for a sustainable future. Renew Sustain Energy Rev. 2008;12(3):593-661.

20. Dincer I, Kanoglu M, Rosen MA. Role of exergy in increasing efficiency and sustainability and reducing environmental impact. Energy Policy. 2008;36(1):128-37.

21. Wall G, Banhatti DG. Exergy: A Useful Concept for Ecology and Sustainability. In Knowledge Systems of Societies for Adaptation and Mitigation of Impacts of Climate Change. 2013: 477-488.

22. Rosen MA, Dincer I, Kanoglu M. Role of exergy in increasing efficiency and sustainability and reducing environmental impact. Energy policy. 2008;36(1):128-37.

22. Rosen MA, Dincer I, Kanoglu M. Role of exergy in increasing efficiency and sustainability and reducing environmental impact. Energy policy. 2008;36(1):128-37.

23. Dietz S, Neumayer E. Weak and strong sustainability in the SEEA: Concepts and measurement. Ecological economics. 2007;61(4):617-26.

24. UNSTATS. System of Environmental-Economic Accounting 2012 Experimental Ecosystem Accounting [Internet]. 2014. Disponible en: http://ec.europa.eu/eurostat/documents/3859598/6925551/KS-05-14-103-EN-N.pdf 